Arteterapia. Papeles de arteterapia y educación para inclusión social ISSN: $1886-6190$

\title{
ConcentrArte: una propuesta de intervención para niños venezolanos diagnosticados con Trastorno de Déficit de Atención
}

\author{
Jhonny De Vasconcelos De Freitas ${ }^{1}$; Marilex Pérez Venegas ${ }^{2}$
}

Recibido: 31 de marzo de 2016 / Aceptado: 14 de julio de 2016

Resumen. CocentrArte es el producto de una investigación cuyo propósito fue diseñar un programa de intervención arteterapéutico para niños venezolanos entre siete y ocho años de edad con diagnóstico de Trastorno por Déficit de Atención con Hiperactividad (TDAH). El resultado es un modelo que involucra a los niños con sus adultos significativos mediante un tratamiento bimodal casa-sesión. Contempla actividades artísticas como medio de expresión del mundo interno del infante y ejercicios de estimulación cognitiva de la atención a estímulos visuales y auditivos. De esta forma, se logró agrupar el terreno arteterapéutico, psicólogico y neuropsicológico.

Palabras clave: Arteterapia; TDAH; Psicología; Neuropsicología; Escolares Venezolanos.

\section{[en] ConcentrArte: an intervention proposal for Venezuelan children diagnosed with Attention Deficit Disorder}

\begin{abstract}
ConcentrArte is a product of an investigation which purpose was focused in designing an intervention art therapy program for venezuelan between seven and eight years old children, whose diagnosis was attention-deficit hyperactivity disorder (ADHD). As a result, children were involved with their significant adults by a bimodal home-session treatment. The program contained a variety of art activities as a way to express inner self emotions and cognitive stimulation exercises for auditive and visual attention. ConcentrArte was a project that gathered psychological, art therapy and neuropsychological fields.
\end{abstract}

Keywords: art therapy, ADHD, psychology, neuropsychology, venezuelan children.

Sumario. 1. Introducción; 2. Arteterapia y Déficit de Atención; 3. Arteterapia como corriente terapéutica: Perspectiva Dinámica; 4. Procesos Cognitivos subyacentes en la Creación Plástica; 5. Estimulación Cognitiva; 6. Diseño del programa ConcentrArte; 7. Metodología; 8. Procedimiento e instrumentos de medición y evaluación; 9. Análisis Cuantitativo de Resultados; 10. Análisis Cualitativos de Resultados; 11 Consideraciones finales; 12. Referencias Bibliográficas.

Cómo citar: De Vasconcelos De Freitas, J.; Pérez Venegas, M. (2016) ConcentrArte: una propuesta de intervención para niños venezolanos diagnosticados con Trastorno de Déficit de Atención, en Arteterapia. Papeles de arteterapia y educación para inclusión social 11, 293-307.

$1 \quad$ Universidad Central de Venezuela

E-mail: artepsique@gmail.com

2 Universidad Central de Venezuela

E-mail: mperezve@ucab.edu.ve 


\section{Introducción}

El TDAH es una patología que de acuerdo con el Manual Diagnóstico y Estadístico de los Trastornos Mentales en su versión cuarta, texto revisado (DSM-IV-TR), que alcanza una prevalencia de un 3 al $5 \%$ de la población infantil en edad escolar (APA, 2000). Actualmente en Venezuela no se conoce con exactitud esta cifra, pero para el año 2002 se estimó un predominio entre el 7 y $10 \%$ de la población, lo cual es considerado un nivel elevado para un trastorno en edad temprana (Montiel y Montiel, 2007).

Acorde con estas cifras, esta patología se convierte en un problema de salud pública, debido a su alta prevalencia y tendencia a convertirse en un proceso crónico y de grandes secuelas en la vida escolar, anímica y social de quien la padece.

La ciencia médica y la psicología han intentado dar respuesta ante tal problemática y se han establecido algunas modalidades de intervención. Entre ellas se encuentran el tratamiento farmacológico, el psicológico en sus diversas modalidades y el terreno de la intervención neuropsicológica (Montiel y Montiel, 2007).

También, han surgido desde otras disciplinas algunas propuestas de tratamiento, entre ellas la terapia ocupacional y la musicoterapia, el Arteterapia para mencionar algunas. Esta última, usada en parte porque "Dentro de sus objetivos destaca la potencialización de las funciones cognitivas fundamentales como: atención, memoria, funciones ejecutivas y coordinación" Gallardo-Saavedra, Padrón-García, Martínez-Waldo, Barragán-Campos, Passaye, García \& Aguilar-Castañeda, 2010, p. 79).

Debido a los numerosos beneficios del arteterapia, la misma ha sido aplicada por diversos autores en pacientes con trastornos cognitivos como la demencia, la esquizofrenia y el TDAH (Rodríguez, 2005; Safran, 2002). Estas intervenciones han sido acompañadas por la neurociencia y la neuropsicología otorgando una mirada innovadora de cómo el arte puede ayudar a superar dificultades e incrementar la potencialidad del ser humano, además de aportar una mirada integradora entre las distintas modalidades de intervención atendiendo a la persona entendida desde su globalidad.

Por lo tanto, establecer intervenciones que permitan agrupar el terreno psicológico, neuropsicológico y arteterapéutico es uno de los nortes al que quiere apuntar el campo de las psicoterapias expresivas y dentro de ellas el arteterapia. Consecuente con esto, Malchiodi (2012) afirma que el futuro de la investigación debe tratar los efectos de la creación artística sobre la cognición. Al mismo tiempo, que confiere importancia al desarrollo de estrategias que desde la unión de lo terapéutico, el estudio del cerebro y sus funciones permitan una mejor comprensión del ser humano como un todo, es decir en sus componentes psicológicos, sociales y biológicos.

De esta forma, ConcentrArte surge como propuesta de la congregación del arteterapia, la psicología clínica y la neuropsicología en el contexto venezolano y se concibe como una modalidad de tratamiento para el TDAH adaptada a las necesidades y especificaciones psicológicas y cognitivas de estos infantes. Asimismo, pretende posibilitar el tratamiento en etapas iniciales del diagnóstico 
con la contingencia de reducir los efectos consecuentes en el rendimiento académico, emocional y social del niño diagnosticado.

\section{Arteterapia y Déficit de Atención}

En la literatura sobre arteterapia en contextos europeos y norteamericanos se manifiesta que los enfoques individuales y estructurados están indicados para los niños con TDAH, debido a que los ayuda en el auto-manejo de su comportamiento y en el aumento de la autoestima (Malchiodi, 2012).

Por su parte, Safran (2002) señala que los grupos de arteterapia fungen como un buen medio para capturar la intensa experiencia que viven, pues el arte permite el reencuentro con los sentimientos y pensamientos una y otra vez, facilitando así su asimilación. De esta forma, las producciones o piezas realizadas actúan como un punto de referencia para el paciente desde el mismo momento del diagnóstico y lo acompaña durante el tratamiento, de manera que pueda percibir su propio recorrido.

También posibilita la estimulación física y sensorial y permite a los niños con síntomas impulsivos estar más tranquilos pues lo ritualista y rutinario de esta modalidad les sirve como contención.

Igualmente, se suscriben cambios cognitivos y así lo señalan Gallardo-Saavedra et al. (2010), quienes observaron mejoras significativas en un grupo de 13 escolares en la comprensión verbal, razonamiento perceptivo y velocidad de procesamiento luego de una experiencia arteterapéutica.

\section{Arteterapia como Corriente Terapéutica: Perspectiva Dinámica}

Desarrollos de los postulados psicoanalíticos permean los antecedentes teóricos y técnicos del arteterapia. En relación al primer aspecto, Freud asevera que existe una cierta flexibilidad en la represión lo que facilita el proceso creativo en el artista, el cual pareciera tener una mayor conexión con el inconsciente y funcionar bajos los principios del proceso primario (Guimón, 2008; López y Martínez, 2006).

Por su parte, Fiorini (2006) desarrolla su teoría del psiquismo creador, definiéndola como "un sistema que se activa y desarrolla en el tiempo mediante la puesta en marcha y resolución-culminación de procesos de orden creador" (p. 12). Para el autor existe un espacio en el psiquismo para lo posible en torno al cual se ordena la creación como proyecto, tomando en consideración los postulados de Winnicott en su teoría, y su obra Realidad y Juego.

En este tipo de pensamiento denominado proceso terciario, el autor establece una tópica en donde distingue tres lugares; lo dado, lo imposible y lo posible. Las formas dadas al sujeto se desorganizan y funda nuevas alternativas que pueden converger y divergir a la vez. El pensamiento creador debe hacerlas resonar entre sí. Finalmente el producto conformado es un objeto con diversas significaciones y temporalidades.

Otro de los postulados relevantes, señala como la realidad psíquica (impulsos y fantasías inconscientes) se plasman en una obra de arte. A través del dibujo, la pintura, o cualquier otra manifestación, se pueden trabajar o elaborar elementos 
traumáticos debido a que tales técnicas se prestan para la compulsión a la repetición y por ende su procesamiento (Levin, 2005).

López (2004) señala que la posibilidad comunicativa que facilita el arte se encuentra ligada a la catarsis como herramienta para acceder al inconsciente. Por su parte, Kramer (2000) asevera que el artista reemplaza el impulso de actuar sus fantasías mediante el acto de crear figuras visuales, dado a que la experiencia gráfica tiene lugar en un mundo simbólico.

En relación a los aspectos técnicos, es necesario destacar la función que realiza la transferencia dentro de la terapia artística, la cual es entendida como "la colección de los impulsos y las fantasías del paciente dirigidos a el terapeuta en el transcurso de la actividad creadora" (McMurray y Schwartz, 1998, p.32). Partiendo del mismo principio que se da en el psicoanálisis y en las terapias derivadas de él. Ahora bien, en el arteterapia, el producto artístico se convierte en el contendor de la interpretación, sin necesidad del uso de la palabra (Carles, 2012).

En otro orden de ideas es común que se utilicen algunas estrategias dentro de la estructuración de las actividades. Tal es el caso del uso del juego del garabateo de Winnicott y el uso de los mándalas desarrollado por Jung, por mencionar los más relevantes (López y Martínez, 2006).

\section{Procesos Cognitivos subyacentes en la Creación Plástica}

Durante décadas, algunos autores se han dedicado a explicar cómo la sola actividad artística, impulsa las regiones corticales y redes neuronales relacionadas con la expresión emocional y el placer. Asimismo, las artes visuales estimulan las áreas visuales del lenguaje, incitan al cerebro a reconocer imágenes, a poderlas nombrarlas y a conseguir crear o reproducirlas, aunque sea a través del reaprendizaje o del modelado de nuevas conductas (Rodríguez, 2005).

Por su parte, Lusebrink (2004) señala que el procesamiento de la información visual se ve estimulado pues existen áreas como la corteza visual primaria del lóbulo occipital que responden a la textura y el color de estímulos visuales. Mientras que la corteza visual secundaria del temporal responde a las características de forma y color. La respuesta a estímulos figurativos activa de forma adicional el hipocampo y el lóbulo temporal inferior derecho.

Según Rodríguez (2005) al activarse los circuitos corticobasales se pone en juego la capacidad de atender y concentrarse. Consecuentemente se inicia el funcionamiento de las habilidades visoespaciales, visoperceptivas y visoconstructivas, función ejecutiva y las habilidades psicomotrices.

García (2011) afirma que pintar "implica trabajar la percepción de formas, la diferenciación figura-fondo, la percepción del color, la organización espacial de los elementos, y la coordinación óculo-manual necesaria para la visoconstrucción" (p. 17). También, declara que pone en juego habilidades como organizar, seleccionar y secuenciar, pues debe realizar una serie de acciones que implican un antes y un después, con lo cual se activa la funcionalidad de las estructuras prefrontales. Finalmente, no deja de lado la habilidad de resolver problemas prácticos, así como la motricidad fina y gruesa.

Específicamente Lusenbrick (2004), comenta que según el tipo de material o técnica que se utilice en la sesión pueden estimularse diferentes procesos 
cognitivos y motores. Mediante la arena, el agua, las semillas, rocas y la pintura de dedos, puede activarse el sistema sensorial y la capacidad motora. Por su parte, el trabajo en tres dimensiones, (bloques de madera, arcilla, plastilina, etc.) se presta para la exploración del espacio y la retroalimentación háptica, mientras que el uso del collage ayuda en la resolución de problemas y en el fomento del pensamiento conceptual y abstracto.

Asimismo, el sentido del tacto es de especial interés en la sesión de arteterapia, pues activa los sentidos cutáneos que responden a la presión, vibración y temperatura lo cual implica percepción y movimiento. A la vez que se activan las emociones porque la amígdala recibe información de la corteza somatosensorial primaria y también de la corteza occipital. De esta forma, la actividad creadora estará relacionada con rasgos determinados de personalidad y la activación de áreas cerebrales específicas que permiten al individuo creativo comprender, desarrollar y expresar de manera sistemática relaciones novedosas y manejar un pensamiento divergente.

\section{Estimulación Cognitiva}

García (2011) entiende la estimulación como toda tarea dirigida a mejorar el rendimiento de procesos cognitivos. Debido a la potencialidad que ofrece puede ser aplicada en cualquier individuo con el fin de desplegar y potenciar su funcionamiento. Está enmarcado en el concepto de neuroplasticidad, es decir la capacidad de generación de nuevas conexiones sinápticas y en la idea de que el entorno produce cambios estructurales en el cerebro (sobre todo a nivel cortical), mejoras a nivel neuroquímico, en el tamaño de los contactos sinápticos y en las dendritas.

Existen algunos principios que subyacen al proceso de estimulación, el primero es involucrar a los familiares significativos del paciente y a los profesionales de la salud como arteterapeutas, psicólogos, neurólogo, terapeutas ocupacionales, entre otros. En segundo lugar, es necesario considerar las metas o aspectos funcionales más relevantes a estimular, es decir, tomar en cuenta aquel proceso cognitivo que va a producir mejoras en la funcionalidad del sujeto. A seguir, no se debe dejar de lado los aspectos afectivos o emocionales de la persona y una vez en marcha la intervención, es indispensable la valoración constante y rutinaria de su eficiencia (Cueva y Quijano, 2008).

Otro punto importante a destacar en el abordaje en el estilo de actividades que se pueden utilizar, García (2011) divide los abordajes en tres formas de intervención, en primer lugar ubica las estructuradas entendidas como aquellas actividades organizadas y con objetivos específicos; así el paciente realizará una serie de ejercicios de manera repetida que comiencen con tareas sencillas y vayan aumentando progresivamente en su dificultad mediante la utilización de guías o ayudas externas al sujeto.

En segundo lugar, se encuentran las de tipo informal, las cuales incluyen tareas de la vida cotidiana, recreación y ocupacionales, que a pesar de no ser formalmente estrategias cognitivas si implican un entrenamiento. Finalmente, aquellas en las que de forma mixta se entrecruzan las estructuradas con las informales. 


\section{Diseño del Programa ConcentrArte}

Tras la exhaustiva revisión de la literatura especializada y ante la necesidad de ofrecer recursos que promuevan mejoras en niños venezolanos con TDAH en edades comprendidas entre 7 y 8 años se diseña el programa ConcentrArte, intervención arteterapéutica que considera los procesos cognitivos subyacente a la creación plástica y se ajusta a la realidad psicosocial en la que se produce. Posteriormente, es sometido al proceso de validación por jueces expertos en el área de desarrollo infantil, arteterapia, programas de intervención y se realizan los ajustes correspondientes.

El resultado es un modelo que involucra a los niños diagnosticados y a sus adultos significativos, mediante un tratamiento bimodal casa-sesión. Para el hogar se establece un libro de estimulación cognitiva de la atención a estímulos visuales y auditivos, partiendo de que este proceso mejora las capacidades mentales, y tiene mayor beneficio si se junta con el quehacer plástico. El material diseñado cuenta con una serie de ejercicios artísticos que deben ser realizados dos veces a la semana por el niño en compañía de un adulto. Estas asignaciones son guiadas por un manual para padres que incluye las especificaciones en su acompañamiento y recomendaciones para la crianza de niños con TDAH.

Paralelamente, en un encuadre terapéutico se brinda un ambiente estructurado (dos sesiones semanales) de forma rutinaria tanto en su inicio, desarrollo y cierre. Para el comienzo se utiliza una dinámica de saludo y compartir del estado anímico de los participantes. El desarrollo contiene una actividad de naturaleza cognitiva (visual o auditiva), otra de expresión emocional mediante el arte y una tarea de retroalimentación (atenciómetro) sobre el nivel de atención del niño.

La estructura está diseñada en fases (asesoramiento, inicial, media y final), además, se enfatiza en brindar un espacio creativo, estructurado y rutinario, bajo una modalidad grupal que funciona con reglas básicas de convivencia (cariño y respeto para los involucrados en la sesión y el seguimiento de instrucciones), asegurando así un espacio seguro con libertad para expresar emociones.

En el proceso de creación plástica se inducen y activan las diferentes modalidades sensoriales y motoras colocando estímulos evocativos (táctiles, sonoros y visuales) al inicio del mismo, ofreciendo medios sólidos y de mayor control por parte de los participantes (lápices de grafito y colores, marcadores punta fina, plastilina dura y collage) en la ejecución de la producción, con el fin de reforzar procesos de orden cognitivo subyacentes en el quehacer plástico.

El cierre de las sesiones se toma en cuenta el triángulo arteterapéutico, es decir, se establece un puente de diálogo entre la producción, el paciente y los terapeutas como un elemento más que permite la retroalimentación. Post sesión se recoge un registro por parte de los terapeutas que incluye la observación de aspectos propios de la patología: tiempo de reacción como medida de impulsividad cuando es inmediato, seguimiento de instrucciones como indicador atencional, tolerancia a la frustración como signo de manejo emocional y expansividad comportamental como señal de hiperactividad.

El énfasis que poseen las actividades en cuanto a la atención visual y auditiva son coherentes con lo planteado por Cueva y Quijano (2008) quienes enuncian a la atención como proceso básico que incide sobre los demás componentes cognitivos 
de manera que es determinante y esencial para cualquier actividad humana y más si se trata de una afección neuropsicológica como el TDAH.

De esta forma, la estructuración de las actividades cognitivas, tanto en el cuaderno para el hogar como en las llevadas a cabo en sesión, obedecen a principios neuropsicológicos y fueron diseñadas tomando en cuenta:

a) Con respecto a la cantidad se plantea un ejercicio por sesión con una duración no mayor de 10 minutos aproximadamente para asegurar que no resulten agobiantes al niño.

b) La dificultad es sistemática y secuencial.

c) Las instrucciones (escritas y orales) son claras y precisas evitando cualquier ambigüedad o término técnico desconocido para los involucrados en la intervención. Al mismo tiempo se asegura que estos elementos estén acordes a la edad, capacidad evolutiva y emocional de los participantes tomando como guía el libro de Secuencias de Desarrollo Infantil Integral (León de Viloria, 2011).

d) El ambiente en que se realiza debe permanecer libre de distracción u excesiva estimulación con un clima de cordialidad y respeto hacia todos los involucrados en el programa (participantes, representantes, docentes, terapeutas y materiales).

Los autores del programa resaltan el énfasis en el uso de la retroalimentación (parte del proceso de estimulación cognitiva), como potenciador de la capacidad de autorregulación, un elemento reforzador positivo de los logros y aciertos en las actividades propuestas para el infante. En concordancia a estas consideraciones se diseña el atenciómetro como una tarea de valoración subjetiva de la ejecución de la atención del niño al final de la actividad realizada, esta apreciación es completada por los niños, padres y terapeutas al final de cada actividad del programa tanto en casa como en sesión.

\section{Metodología}

La presente investigación se llevó a cabo con el objetivo de diseñar un programa de intervención arteterapéutico sobre la atención visual y auditiva y la hiperactividad e impulsividad de niños diagnosticados con TDAH. Para ello se llevó a cabo una investigación cuasi experimental con un grupo natural, a saber, niños con TDAH.

Se aplicó un diseño pre y post test de un sólo grupo. Primero se estableció una línea base, se produce la aplicó el programa de intervención a 6 niños con diagnóstico con TADH y se hizo una nueva medición al finalizar. Además se realizó mediciones semanales con el fin de conocer la evolución de la sintomatología y de captar datos de interés para la investigación, de manera que se realizó un diseño mixto dado que para asegurar una mayor comprensión de los resultados se llevó a cabo un análisis cualitativo de las variables medidas.

Se tomaron medidas pre y post ConcentrArte de la atención visual, atención auditiva, tendencia a la inatención, hiperactividad e impulsividad. Dentro de las variables controladas se tuvo que las sesiones del programa se realizan en un mismo espacio, con condiciones apropiadas y constantes, es decir, la iluminación y la ventilación natural, así como el tiempo, lugar y los materiales de trabajo. Por otra parte, se toma en cuenta participantes varones entre 7 y 8 años de edad, con diagnóstico de TDAH. Se procuró mantener un nivel similar en las instrucciones y actividades de manera que involucre retos biológicos, psicológicos y sociales 
similares, junto a la diversidad de motivaciones e intereses inherentes a los mismos asociados a su momento evolutivo.

Descriptivamente la muestra estuvo conformada por 5 varones con el diagnóstico de TDAH, cuatro de ellos de tipo inatento y uno combinado, en edades comprendidas entre 7 años y 0 meses y 8 años y 10 meses, un nivel socioeconómico entre el estrato III y V, dos de ellos cursando 2do grado y tres niños en 3er grado de educación básica, donde dos compartían salón.

En cuanto a los antecedentes se obtuvo, que ninguno de los participantes hubiera sido sometido a intervenciones psicológicas, y sólo uno de ellos presentaba tratamiento psicofarmacológico tradicional. Todos ellos provenían de familias disfuncionales, en específico se caracterizaban por familia monoparental con conflictividad marcada para llevar la crianza conjunta, lo que conllevaba a un ambiente de negligencia y abandono, poco estructurado, con dificultades para establecer límites o normas claras y consistentes en el tiempo. Brindando un contexto familiar poco contenedor afectivamente.

Una vez concluido el estudio sólo se tomó en cuenta los datos correspondientes a 5 de los 6 participantes, ya que uno de estos faltó al $25 \%$ del tratamiento y no se obtuvo la medición postest.

\section{Procedimiento e instrumentos de medición y evaluación}

a. Fase de diseño: Se diseñó el Programa de Intervención teniendo presente los criterios teóricos y práctico en arteterapia, la problemática descrita del trastorno y los aspectos concerniente al desarrollo infantil de los participantes.

b. Fase de validación: Se contactó a 5 jueces expertos solicitando su colaboración, entregando un formato para recoger sus apreciaciones, realizando los ajustes correspondientes antes de la aplicación del programa.

c. Fase muestral: se contactó una institución educativa regular a la cual se le presentó el programa al equipo directivo y psicopedagógico conformado por una psicóloga, y varias psicopedagogas y docentes especialistas.

d. Fase de identificación: una vez aprobado la aplicación, se identificaron junto con la psicóloga de la unidad psicopedagógica, a los niños que cumplían los criterios de inclusión para ser parte de la muestra seguidamente se convocaron a los representantes de 10 posibles participantes, de los cuales asistieron 5 y en una segunda convocatoria se reclutó uno más, para un total de 6 niños.

e. Fase de autorización: Se obtuvo el consecutivo permiso del representante del sujeto escogido, considerando además las normas en relación al consentimiento informado, mantenimiento de la intimidad y la confidencialidad y de los artículos de la LOPNA referentes al resguardo de los niños que participan en una actividad con fines de investigación.

f. Fase de Pre-Test: Se realizó la primera medición con el fin de obtener la línea base. Los instrumentos utilizados fueron: Las subescalas de atención auditiva (Dígitos en progresión y Dígitos en Regresión) y visual (Cancelación de Letras y Cancelación de Dibujos) de la Batería de Evaluación Neuropsicológica Infantil (ENI) elaborada por Ardila et al. (2005). Las cuales son utilizadas en Investigaciones como las de Bará et al. (2003) y Montoya, Varela \& Dussán (2011) para la detección de déficit específicos en los procesos cognitivo de la atención. 
Asimismo, se empleó la Escala de Clasificación Diferencial del TDAH (ESCADITA) elaborada por Rodríguez, et al., (1997), la cual es una escala tipo likert de reporte para el hogar y la escuela, construida en una muestra 714 niños caraqueños entre 6 y 12 años de edad, la cual mide los signos y síntomas comportamentales propios del TADH, a saber, inatención, hiperactividad e impulsividad, así como, la presencia de los factores que mayormente son comórbidos al trastorno (TOD y DA en lectura, escritura y matemáticas).

Esta escala incluye una versión para el hogar $($ alpha crombach $=.93)$ y otra para la escuela (alpha cromba Esta escala se consideró la más pertinente dado que fue construida en el contexto venenezolano, lo cual permitió una adecuada aproximación a las variables del presente estudio.

\section{Análisis Cuantitativos de los Resultados}

La información fue procesada por el programa SPSS 20.0 para Windows (C) con el objetivo de poner a prueba las hipótesis sobre la efectividad del programa se llevaron a cabo análisis estadísticos del tipo descriptivo y de diferencia de medias.

En primer lugar, al realizar un análisis comparativo de las medidas descriptivas del Pre y Post Test se obtuvo que en cuanto a la atención visual el grupo, específicamente, en la prueba de cancelación de dibujos la ejecución fue homogénea mostrando una mejoría en el post test, pasando de promedio bajo a promedio. En cuanto a cancelación de letras la ejecución fue homogénea y se mantuvo en promedio bajo hasta el final del tratamiento.

Con respecto a la atención auditiva el grupo mostró una ejecución homogénea, específicamente en la prueba de dígitos en progresión pasó de promedio bajo a promedio. Mientras que en la prueba de dígitos en regresión, la misma alcanzó puntuaciones de promedio alto, mostrando así una mejoría en ambos casos.

En segundo lugar, cuando se comparó el reporte (ESCADITA, versión hogar) de los representantes del grupo en cuanto a los factores propios del TDAH (inatención, hiperactividad e impulsividad) se observó que si bien se mantiene la presencia de cada factor, existe una homogeneización en la presentación de los síntomas al final del tratamiento. En relación a la sintomatología comórbida (TOD y DA) en el grupo se observó la presencia de ambas, no obstante, el factor DA Matemática fue la única que resultó en una ejecución homogénea al final del tratamiento, los demás factores se mantuvieron heterogéneos.

Asimismo, cuando se comparó el reporte (ESCADITA, versión escuela) de los docentes del grupo para los factores propios del TDAH (inatención, hiperactividad e impulsividad) se observó que se mantiene la presencia de cada factor, especialmente, la inatención no varió en su ejecución heterogénea, mientras que para los demás factores (hiperactividad, impulsividad, TOD, y DA) la ejecución varió de heterogénea a homogénea.

En tercer lugar, para poner a prueba las hipótesis en relación a la efectividad del tratamiento, se evaluó si habían diferencias significativas entre las medias pre y post tratamiento en cuanto a las variables de atención auditiva, atención visual y por otro lado las comportamentales: inatención, hiperactividad, impulsividad, TOD, DA en lectura, escritura y matemática tanto en el hogar como en la escuela por medio de la Prueba de los rangos de Wilcoxon, siendo la más indicada en la 
literatura para muestras relacionadas, dado que sus resultados son tan válidos como una $t$ de Student para muestras en las que se puede asegurar su normalidad.

De esta forma, la mayoría de las hipótesis planteadas resultaron estadísticamente no significativa, específicamente, para cancelación de dibujos $(Z=-1,48 ; p=.138)$, cancelación de letras $(Z=-1,633 ; p=.102)$, dígitos en progresión $(Z=-1,342 ; p=.18)$, en relación a las variables de la ESCADITA versión hogar se obtuvo que: inatención $(Z=-1,483 ; p=.138)$, hiperactividad $(Z=-1,214 ; p=.225)$, impulsividad $(Z=-0,365 ; p=.715)$, TOD $(Z=-0,677 ; p=.498)$, D.A. Lectura $(Z=$ $1,355 ; p=.176)$, D.A. Escritura $(Z=-1,49 ; p=.136)$, D.A. Matemáticas $(Z=-1,826$; $\mathrm{p}=.68)$. Ahora bien, en relación a la versión escuela los resultados indicaron que: inatención $(Z=-1,153 ; p=.8)$, hiperactividad $(Z=-1,084 ; p=.279)$, TOD $(Z=0$; $p=1.000)$, D.A. Lectura $(Z=-, 542 ; p=.588)$, D.A. Escritura $(Z=-1,214 ; p=.225)$, D.A. Matemáticas $(Z=-0,552 ; p=.581)$. Por otra parte, solo dos variables resultaron estadísticamente significativas, específicamente, dígitos en regresión $(Z=-, 206$; $\mathrm{p}=.039)$, e impulsividad para la escuela $(Z=-2,032 ; \mathrm{p}=.042)$.

\section{Análisis Cualitativo de los Resultados}

\section{Sobre el Desarrollo del Programa}

Una vez diseñada la versión piloto del programa ConcentrArte el mismo fue entregado a 5 jueces expertos en el área; de los cuales sólo cuatro de ellos respondieron, dos especialista en programas de intervención escolar e infantil y dos arteterapeutas. En base a las observaciones realizadas se establecieron los cambios pertinentes en relación a tres aspectos fundamentales, a saber, la restricción de los materiales que en principio se pretendían ofrecer, la reducción del número de sesiones, la adecuación del lenguaje al momento evolutivo de los sujetos participantes en las instrucciones dentro de la sesión.

En cuanto a la estructuración del programa y de cada una de las sesiones, el diseño de las actividades propuestas, y su adecuación al nivel evolutivo de los participantes con el diagnostico TDAH, así como de su coherencia con los objetivos tanto del programa, como por sesión, aspectos que fueron valorados positivamente unánimemente por los jueces.

Asimismo el cuaderno de estimulación cognitiva que forma parte del programa ConcentrArte fue sometido a validación con los mismos criterios antes mencionados. El resultado de este proceso arrojó la necesidad de adecuar el lenguaje utilizado en las instrucciones al nivel evolutivo de los participantes. El manual para padres fue considerado adecuado y pertinente en la totalidad de los aspectos.

Así llegado el momento de la aplicación se tomaron en cuenta las consideraciones teóricas y prácticas en relación al setting arteterapéutico, en particular esta aplicación ocurrió en un salón que contaba con las condiciones relacionadas con las condiciones de luz, ventilación y libre de ruido y distracciones en el ambiente.

El desarrollo de las sesiones se produjo acorde a las especificaciones descritas en el programa, asimismo se cumplieron los objetivos sesión tras sesión. Ahora bien, dada la dinámica que se genera en un grupo de arteterapia y los síntomas de 
impulsividad e hiperactividad propios del TDAH, durante el inicio y cierre de las actividades las mismas se llevaron a cabo de manera entorpecida.

Por otra parte, fue necesario realizar ajustes, en la mitad del programa, haciendo énfasis en solo dos normas particulares, a saber: a) seguir instrucciones y b) cariño y respeto tanto para los involucrados (terapeutas y participantes) como para los materiales y el espacio donde se realizaba la sesión. Adicionalmente, se introdujo una técnica de modificación de conducta, específicamente, la economía de fichas para reforzar el cumplimiento de las normas.

\section{Sobre las Producciones Plásticas}

En primer lugar, tal como se describe en el programa, las producciones plásticas de los sujetos se llevaron a cabo en tres fases y para su análisis se establecieron los siguientes criterios: la técnica, el uso del espacio, el tipo de trabajo, la calidad de la línea y la descarga motora.

De esta forma, la técnica hace alusión al empleo o no de la modalidad plástica que se propuso para la actividad, el uso del espacio está relacionado con el manejo que hace el sujeto del área de soporte (hoja, papel, cartulina etc), el tipo de trabajo se entiende como la naturaleza figurativa o no de la producción, la calidad de la línea a su vez, refiere al tipo de trazado con el cual se ejecuta la obra y finalmente, la descarga motora, describe la impulsividad observada en el proceso creativo.

A modo de resumen, los resultados evidenciaron que todos los participantes emplearon la modalidad plástica sugerida para cada sesión, 3 sujetos mostraron expansividad en las primeras dos fases del tratamiento, 4 sujetos generaron una producción de tipo figurativo, lo cual es esperado de acuerdo a su nivel evolutivo, no obstante, en cuanto a la calidad de la línea la tendencia del grupo ( 3 de 5 sujetos) fue un trazo descoordinado y descuidado, mientras que la descarga motora tendió a la ausencia en tres de los sujetos durante el programa y en dos de ellos solo en la fase final.

Complementando las actividades artísticas, se ejecutaron ejercicios de naturaleza cognitiva con especial énfasis en procesos atencionales tanto visuales como auditivos, para llevar acabo su análisis se establecieron criterios dicotómicos, a saber, el seguimiento de instrucciones y el coloreado tanto en la sesión arteterapéutica como en el hogar.

En resumen, se observó en cuanto al seguimiento de instrucciones que en sesión los cinco participante lograron cumplirlas en todo momento, mientras en el coloreado presentaron mayor variabilidad, es decir, no se observó un patrón en la ejecución del grupo, específicamente, dos sujetos presentaron durante el tratamiento un coloreado descuidado y descoordinado, sin embargo, el coloreado cuidadoso y coordinado estuvo presente para un sujeto solo en la última fase, en otro sujeto solo en la dos primeras fases, y el último sujeto lo logró en todo momento.

Ahora bien, en relación a estas mismas medidas en casa, se observó que cuatro de los participantes presentaron un coloreado descoordinado y descuidado en todas las fases del tratamiento y el último sujeto solo lo presentó en la última fase. Mientras que para el seguimiento de instrucciones se observó variabilidad en la ejecución del grupo, específicamente, dos sujetos alcanzaron el seguimiento solo 
en la primera fase, uno lo alcanzó solo en las dos primeras fases, y por último, dos sujetos alcanzaron el seguimiento en todas las fases.

\section{Sobre los atenciómetros}

Como parte de las valoraciones de los procesos cognitivos así como procurando estimular la automodulación de los comportamientos y afectos, así como introduciendo un elemento de retroalimentación tanto de los terapeutas (en sesión) como de los representantes (en casa) hacia los participantes, se empleó el atenciómetro, tal y como se describe en el programa. Siendo una escala continua que va del 1 al 5, en donde a mayor puntuación es indicador de una mayor valoración subjetiva de la atención. Cabe destacar que dado a la naturaleza de cierre de procesos arteterapéuticos en la sesión 12 no se llevó a cabo la recolección de los atenciómetros.

En síntesis, se observó que cuatro de los participantes tanto en sesión como en el hogar presentaron autovaloraciones entre buena a excelente, a excepción, de uno de ellos que mostró el reconocimiento de la variabilidad de su capacidad atencional durante las actividades. Por su parte, tanto terapeutas como representantes indicaron la presencia de mayor variación en el desempeño atencional. Los terapeutas marcaron puntuaciones entre muy poco a excelente, mientras que en casa, el representante, aunque fueron capaces de indicar la misma variabilidad en la capacidad atencional, estos lo hicieron con puntuaciones entre regular a excelente.

\section{Consideraciones finales}

El TDAH es uno de los trastornos con mayor prevalencia en la infancia y está caracterizado por presentar síntomas de orden comportamental (impulsividad e hiperactividad) y signos neuropsicológicos (inatención, menoscabo en la función ejecutiva). Que pueden ir acompañados con dificultades en el aprendizaje y trastorno oposicionista desafiante, lo cual conlleva consecuencias en la adaptación familiar y escolar. Estas características, en conjunto con la variedad de presentación de este síndrome, incrementan la dificultad en el tratamiento de estos pacientes.

Por estas razones, es importante poder realizar aproximaciones que abarquen la complejidad de los síntomas del TDAH y le permitan al individuo el aumento de sus recursos académicos, sociales y familiares. Autores como Barkley (2002), Barkley \& Murphy (2006), Miranda et al., (2010), Safran (2012), Zuliani et al., (2008), enfatizan la importancia de realizar diagnósticos e intervenciones tempranas para evitar un mal pronóstico, y otros especialistas (Jarque-Fernández, 2012; León de Viloria, 2011) señalan la relevancia de incluir en el tratamiento a los adultos significativos y docentes en el caso de los niños.

En este sentido, el arteterapia ha sido una técnica que ha intentado dar respuesta a este cuadro, pero no se ha logrado un consenso en sus hallazgos (GallardoSaavedra et al, 2010; González y López, 2011) y es a partir de esta premisa que se diseña el programa Concentr-Arte, el cual es una intervención arteterapéutica con énfasis en la estimulación cognitiva orientada a mejorar la atención visual y auditiva, hiperactividad e impulsividad de niños diagnosticados con TDAH. 
Dado los resultados de esta investigación se puede concluir que; en primer lugar, en la atención de estímulos visuales y la presencia del factor inatención, no existió una diferencia estadísticamente significativa en la medición cuantitativa, lo cual también se observa en el análisis cualitativo, en donde los ejercicios de estimulación cognitiva en sesión y en casa, presentan un predominio de un coloreado descuidado y sin respeto por los contornos de la imagen, lo cual pudiese ser explicado por la presencia del factor de hiperactividad que genera una dificultad de mostrar una disposición cuidadosa al momento de ejecutar las actividades; esto se confirma en el registro realizado por los facilitadores donde se evidenció un tiempo de reacción inmediato luego de dar la instrucción de trabajo, en la poca focalización al momento de actuar y su fácil distractibilidad con conversaciones recurrente que se mantuvieron todas las sesiones del programa.

En segundo lugar, en cuanto a las variables que resultaron estadísticamente significativas se encuentra la medida de la impulsividad en la escuela, en esta valoración las maestras reportan haber observado un cambio en los síntomas conductuales de los niños luego del tratamiento, lo cual no se presentó en el registro de los cuidadores significativos, estos resultados pudieran explicarse por lo señalado por Henley citado en Kearns y Linn (2004) quien encontró que el ejercicio arteterapéutico, generó cambios en los niños impulsivos, pues lo ritualista y rutinario de esta intervención les sirve como contención, confirmándose en los trabajos de Girloy, (2012), Kearns y Linn, (2004) y Safran (2002). Ahora bien, en relación a la ausencia de significación estadística para este factor en la medición en casa, autores como Gallardo-Saavedra et al. (2010) han tenido experiencias similares. Estos resultados pueden ser explicados por la caracterización disfuncional de la familia que no favorece un ambiente estructurado y rutinario con límites claro como ocurre en la escuela y en el programa ConcentrArte y la percepción que tienen los padres del problema del niño.

Adicionalmente, al realizar un análisis cualitativo de los atenciómetros, que como se señaló anteriormente es una medida subjetiva de la atención orientada a estimular la capacidad moduladora y autoreguladora de la conducta impulsiva e hiperactiva, se evidenció que a lo largo del tratamiento ésta fue cambiando desde una sobrevaloración optima de su rendimiento, hasta llegar a un punto en que algunos participantes se percataban de sus componentes impulsivos, hiperactivos e inatencionales y lo reflejaban en su autovaloración, tal como ocurre en el sujeto 4 . Estos resultados, pudieran estar explicados por lo señalado por Girloy, (2012) y Malchiodi (2012) quien afirma que tratamientos de este tipo favorecen el automanejo del comportamiento y ayuda a los niños impulsivos a ser más calmados y centrados. Y por otra parte, la actividad del atenciómetro implica el modelado o reaprendizaje de nuevos patrones de comportamiento a través de la observación del niño de la valoración de este por parte de sus adultos significantes (terapeutas y representantes), tal como Albillo (2007) cuando señala la función de las artes visuales en la conducta.

En tercer lugar, al analizar las variables que no arrojaron una significación estadística, se encuentran los trastornos con los cuales puede cursarse el TDAH, específicamente las dificultades en el aprendizaje y el trastorno oposicionista desafiante. Los autores de la presente investigación consideran que los resultados no arrojaron diferencia alguna pues el programa no contempla su intervención. 
Además de las variables medidas, esta investigación cuenta con las producciones artísticas de los participantes, las cuales fungen también como indicadores de la sintomatología clínica de los pacientes.

Específicamente en niños con TDAH, López (2004) considera que el acto creador actualiza diversas imágenes y éstas tienen la facultad de ampliar la conciencia en cuanto a los conocimientos, las ideas, los pensamientos y los sentimientos. En este sentido, durante las sesiones arteterapéuticas, mientras el paciente desarrolla su trabajo artístico va expresando su egocentrismo infantil, asimismo, se va desenvolviendo la transferencia en la figura del terapeuta que poco a poco es superada mientras el paciente va elaborando cual es la fuente de su problema (Allen, 1988).

En la presente investigación estos elementos señalados teóricamente se evidencian ya que se pueda observar que la temática desarrollada por cada participante en su obra muestra elementos de su realidad psíquica, de sus vivencias y preocupaciones, por otro lado, con los terapeutas los participantes establecieron un vínculo similar al rol paterno y materno, desplegándose así comportamiento transferenciales y contratransferenciales típicos de una atmósfera familiar, que brindaron un guía en la conducción del tratamiento.

Durante este proceso, el uso de los materiales es otro elemento que permite ver como la asociación libre en la actividad creadora va a contener los impulsos y fantasías (McMurray y Schwartz, 1998), esto se hace notar en la disposición de los participantes al momento de usarlos, la cual en un primer momento obedecía a una voracidad y desbordamiento en la cantidad y calidad en la obra y que posteriormente en la fase final del tratamiento se observa un cambio cualitativo en estos impulsos voraces, que aunque se mantienen no se presentaron en la misma intensidad o forma.

El programa ConcentrArte logró ser un espacio seguro, ameno y con límites claros para niños con TDAH que permite expresar aspectos dolorosos que difícilmente pueden ser comunicados en otros espacios como en el hogar o en la escuela, por la dificultad de traer al presente y nombrar determinados hechos, de índole más dolorosa e incluso traumática, a nivel emocional.

Esta premisa sólo es posible mientras se procure mantener un ambiente contenedor mediante el acto creador, lo cual va a permitir la actualización de diversas imágenes y éstas tendrán la facultad de ampliar la conciencia en cuanto a los conocimientos, las ideas, los pensamientos y los sentimientos de los involucrados.

Por otra parte, procura ser el primer programa venezolano en donde la integración de saberes en arteterapia, psicología clínica y neuropsicología se sumen con el fin de convertirse en una intervención pertinente y beneficiosa para niños entre 7 y 8 años de edad con TDAH.

En este sentido la utilidad de la intervención está también determinada por la significación clínica, en el presente estudio, una intervención dirigida a niños con TDAH, el objetivo consiste en la disminución de la sintomatología de hiperactividad, impulsividad e inatención, mejorando de esta manera la inserción del niño a sus diferentes contextos, a saber, familiar y escolar. Mejoría que fue valorada por las maestras como "alumnos menos impulsivos" al final del programa. 


\section{Referencias bibliográficas}

American Psychiatric Association. (2000) Diagnostic and statistical manual of mental disorders (4⿳亠丷厂 ed.). DSM-IV TR. España, Masson.

Carles, R. (2012). La comprensión en Arteterapia Ponencia presentada en la Jornada de la KACAT en Seoul. Inspira, 2, 57-63.

Cueva, M. \& Quijano, M. (2008) Las alteraciones de la atención y su rehabilitación en trauma craneoencefálico. Pensamiento Psicológico, 4,11,167-181.

Fiorini, H (2006) El psiquismo, teoría y clínica de procesos terciarios. Buenos Aires, Argentina: Nueva Visión.

Gallardo-Saavedra, G., Padrón-García, A.L., Martínez-Waldo, M.C., Barragán-Campos, H.R.,Passaye, E., García- Díaz, R \& Aguilar-Castañeda, E. (2010) El arte terapia como tratamiento del trastorno por déficit de atención en una muestra de escolares mexicanos. Archivos de Neurociencias, 15,2, 77-83.

García, J. (2011) Estimulación Cognitiva. España, Universidad de Murcia.

Guimón, J. (2008). Terapia por el Arte. Cuaderno de Psiquiatría Comunitaria, 8(1), 9-25.

Kramer, E. (2000). Art as Art Therapy. London y Philadelphia: Jessica Kingsley Publishers.

León de Viloria, CH. (2011) Secuencias de desarrollo infantil integral (3era.ed.). Venezuela, Publicaciones UCAB.

Levin, R. (2005). La escena inmóvil. Teoría y clínica psicoanalítica del dibujo. Buenos Aires Argentina: Lugar.

Lusebrink, V. (2004) Expressive Therapies Continuum: Implications for Brain. Art Therapy: Journal of the American Art Therapy Association, 27,4,168-177.

López, B. (2004). Arteterapia: Otra forma de curar. [Versión electrónica]. Revista de Investigación aplicada y experiencia educativas, (10), 101-110

López, J. C. (2012). Estructuras internas y trazos abstractos. Revista de Arte, 1, 5.

López, M. y Martínez, N. (2006). Arteterapia: conocimiento interior a través de la expresión artística. Psicothema, 20(1), 62-70.

Malchiodi, C. (2012) Handbook of Art Therapy. Estados Unidos de América, GuilfordPress.

Mcmurray, M., \& Schwartz, M. (1998). Transference in Art Therapy: A new Outlook. The Arts in Psychotherapy, 25(1), 31-36.

Montiel, N. \& Montiel, B. (2007) Presentación clínica del trastorno por déficit de atenciónhiperactividad como función del género. Investigación Clínica, 48,4, 459 - 468.

Rodríguez, N. (2005) Arteterapia. Talleres de Psicoestimulación a través de las artes. Fundación Uszheimer.

Safran, S.(2002) ArtTherapy and AD DH. Diagnostic and Therapeutic Approaches. London, Jessica Kingsley Publishers.

Winnicott, D. (1968). El juego del Garabateo. Voices: The Art and Science of psychotherapy, 4, 24-43.

Winnicott, D. (1971/2008). Realidad y juego (11 ${ }^{\mathrm{a}}$. Ed). Barcelona, España: Gedisa. 